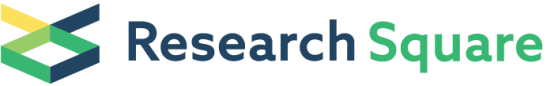 \\ Preprints are preliminary reports that have not undergone peer review. \\ They should not be considered conclusive, used to inform clinical practice, or referenced by the media as validated information.
}

\section{Cost-effectiveness of Anbainuo Plus Methotrexate Compared to Conventional Disease-modifying Antirheumatic Drugs for Rheumatoid Arthritis Patients in China}

\section{Feng Tian}

Department of Rheumatology and Immunology, ZhuZhou Central Hospital, The Affiliated ZhuZhou Hospital XiangYa Medical College CSU

\section{Zhenhua Wen}

Department of Rheumatology and Immunology, ZhuZhou Central Hospital, The Affiliated ZhuZhou Hospital XiangYa Medical College CSU

Jingyang Li ( $\square$ jinyi7139022@163.com )

ZhuZhou Central Hospital, The Affiliated ZhuZhou Hospital XiangYa Medical College CSU

\section{Xiaowen Luo}

Department of Rheumatology and Immunology, ZhuZhou Central Hospital, The Affiliated ZhuZhou Hospital XiangYa Medical Collge

\section{Li Deng}

Department of Rheumatology and Immunology, ZhuZhou Central Hospital, The Affiliated ZhuZhou Hospital XiangYa Medical Collge CSU

\section{Liang Zhang}

Department of Rheumatology and Immunology, ZhuZhou Central Hospital, The Affiliated ZhuZhou XiangYa Medical Collge CSU

\section{Jingyun He}

Department of Rheumatology and Immunology, ZhuZhou Central Hospital, The Affiliated ZhuZhou XiangYa Medical College CSU

\section{Fangling Yao}

Department of Rheumatology and Immunology, ZhuZhou Central Hospital, The Affiliated ZhuZhou Hospital XiangYa Medical College CSU

\section{Zheng Liao}

Department of Rheumatology and Immunology, ZhuZhou Central Hospital, The Affiliated ZhuZhou XiangYa Medical College Csu

\section{Research article}

Keywords: Anbainuo, Methotrexate, Conventional disease-modifying antirheumatic arthritis drugs, Rheumatoid arthritis, Cost-effectiveness

Posted Date: September 21st, 2020 
DOI: https://doi.org/10.21203/rs.3.rs-59486/v1

License: (c) (i) This work is licensed under a Creative Commons Attribution 4.0 International License. Read Full License 


\section{Abstract}

Background: This study aimed to evaluate the cost-effectiveness of anbainuo (ABN) plus methotrexate (MTX) versus conventional disease-modifying antirheumatic drugs (CDMARDs) in Chinese rheumatoid arthritis (RA) patients.

Methods: Ninety RA patients who underwent ABN+MTX (assigned as ABN+MTX group ( $\mathrm{N}=47)$ ) or cDMARDs (assigned as control group $(\mathrm{N}=43)$ ) treatment were analyzed. Disease activity was assessed at baseline $(\mathrm{M} 0), 3^{\text {rd }}$ month (M3), $6^{\text {th }}$ month (M6) and $12^{\text {th }}$ month (M12) after treatment. Drug, other medical, indirect and total costs were calculated. Then pharmacoeconomic analyses were performed with the threshold of cost-effectiveness set as 3 times of the mean gross domestic product per capita in China during the study period.

Results: Treatment response rate was similar, while disease remission and low disease activity rates were increased in ABN+MTX group compared to control group. Drug cost, other medical cost and total cost were higher in $A B N+M T X$ group than control group, while indirect cost was similar between the two groups. Meanwhile, the quality-adjusted life years (QALY) in ABN+MTX group and control group were 0.72 and 0.48 years, respectively. The incremental cost-effectiveness ratios (ICER) of ABN+MTX group compared to control group in total patients, moderate-disease-activity patients and severe-disease-activity patients were $¥ 135486.7, ¥ 146450.4$ and $¥ 124987.2 /$ QALY, respectively, which were all below cost-effectiveness threshold. Further sensitivity analyses revealed that the cost-effectiveness of $A B N+M T X$ versus CDMARDs was relative robust, while among all the indexes, $A B N$ price and HAQ-DI score change for ABN+MTX group affected ICER most.

Conclusions: ABN+MTX treatment presents acceptable cost-effectiveness compared to cDMARDs treatment in Chinese RA patients.

\section{Background}

Rheumatoid arthritis (RA) is one of the most common chronic, inflammatory, autoimmune diseases with a prevalence of $0.5 \%-1 \%$ worldwide and $0.19 \%-0.4 \%$ in China [1, 2]. Clinically, RA patients not only suffer from swelling and pain in both small joints (e.g., metacarpophalangeal) and large joints (e.g., ankle), but also may be affected by extra-articular manifestations such as rheumatoid vasculitis and interstitial lung disease [3]. Meanwhile, RA patients might develop multiple comorbidities (e.g., cardiovascular disease) as a consequence of chronic inflammation [4]. Worse of all, if insufficiently treated, RA will accumulate irreversible joint damage and lead to disability, which greatly affects the quality of life of RA patients and aggravates humanistic as well as economic burden [5].

Tumor necrosis factor (TNF) inhibitors are a vital group of biological disease-modifying antirheumatic drugs (DMARDs), which remarkably suppress the disease progression of RA patients by ameliorating systematic inflammation rapidly, reducing disease activity, suppressing radiology progression to some extent and improving the quality of life of RA patients greatly [6, 7]. However, TNF inhibitors are quite costly, and only a few RA patients in China are willing to pay for them due to the relatively low income of Chinese residents and the need for sustained administration of TNF inhibitors [8, 9]. Therefore, the cost-effectiveness of TNF inhibitors should be investigated, which might help to guide the administration of TNF inhibitors in RA patients in China. 
Anbainuo (ABN), a biosimilar to etanercept (one kind of TNF inhibitor), is a recombinant human TNF-a receptor II: Fc fusion protein developed by local Chinese pharmacology company [10]. According to a phase II trial, $A B N+$ methotrexate (MTX) shows increased treatment efficacy compared to ABN or MTX monotherapy in MTXnaïve RA patients [10]; another phase III trial reveals that ABN+MTX presents good efficacy and tolerance in MTX inadequately-responded RA patients compared to placebo [11]. Thus, $A B N$ is a promising biological agent to treat RA patients in China. Moreover, ABN price is far less than original etanercept, which might enable $A B N$ an available and popular TNF inhibitor in RA patients in China. In order to investigate its cost-effectiveness, our pilot study with a small sample size reveals that $A B N+M T X$ is cost-effective compared to cDMARDs in treating RA patients [12]; however, these results should be further verified with larger populations. Therefore, in this study, we enrolled 55 ABN+MTX-treated patients and 54 cDMARDs-treated patients, and evaluated the treatment efficacy/utility as well as cost during the study, aiming to compare the cost-effectiveness of ABN+MTX versus cDMARDs in Chinese RA patients.

\section{Methods}

\section{Patients}

From March 2015 to October 2018, a total of 109 RA patients who underwent ABN+MTX or conventional diseasemodifying antirheumatic drugs (cDMARDs) treatment at ZhuZhou Central Hospital were consecutively enrolled in this study. The screening criteria were referring to the protocol described in our first-stage study [12]. This study was approved by the Ethics Committee of ZhuZhou Central Hospital (Approved No. 2015-K-05010). All procedures were conducted in accordance with the provisions of the Declaration of Helsinki and Good Clinical Practice guidelines as defined by the International Conference on Harmonization. All patients signed the written informed consents. Besides, the study was registered on the Chinese Clinical Trial Registry (ChiCTR) affiliated to World Health Organization (WHO) (www.chictr.org.cn) with registry number ChiCTR2000032534.

\section{Data collection}

After enrollment, the baseline (M0) characteristics of patients were recorded, which included age, gender, disease duration, tender joint count (TJC), swollen joint count (SJC), C-reactive protein (CRP) level, erythrocyte sedimentation rate (ESR) level, pain visual analogue scale (VAS) score, patient global assessment (PGA) score, physician global assessment (PhGA) score, Health Assessment Questionnaire Disability Index (HAQ-DI) score and history of treatment. And the disease activity of patients was assessed according to disease activity score with 28 joints based on CRP (DAS28-CRP) and disease activity score with 28 joints based on ESR (DAS28-ESR), which were calculated as follows: DAS28-

$\mathrm{CRP}=0.56 * \operatorname{sqrt}(\mathrm{TJC} 28)+0.28 * \operatorname{sqr}(\mathrm{SJC} 28)+0.014 * \mathrm{PGA}+0.36 * \ln (\mathrm{CRP}+1)+0.96, \mathrm{DAS} 28-$

$\mathrm{ESR}=0.56 * \operatorname{sqr}(\mathrm{TJC} 28)+0.28 * \operatorname{sqr}(\mathrm{SJC} 28)+0.014 * \mathrm{PGA}+0.70 * \ln (\mathrm{ESR})$.

\section{Treatment}

After enrollment, 55 patients about to receive $A B N+M T X$ therapy were assigned to $A B N+M T X$ group, and the other 54 patients who scheduled to receive cDMARDs treatment were allocated to control group. The treatment regimens for the two groups were conducted in accordance with the protocol described in our first-stage study 
[12]. In brief, for the $A B N+M T X$ group, $A B N$ and MTX were administrated to the patients as follows: ABN 25 mg twice a week subcutaneously for 24 weeks or ABN 50 mg once a week subcutaneously for 24 weeks, and MTX 10$20 \mathrm{mg}$ once a week orally for 24 weeks. For the control group, all patients received cDMARDs alone or in combination for 24 weeks, and 23 patients received MTX (10-20 mg once a week orally)+leflunomide (10 mg qd), 31 patients received MTX (10-20mg once a week orally)+sulfasalazine ( $1 \mathrm{~g}$ tid orally)+hydroxychloroquine (400 mg qd).

\section{Follow-up}

After initiation of treatment, regular follow-up was conducted for the patients, and the TJC, SJC, ESR level, CRP level, pain VAS score, PGA score, PhGA score, and HAQ-DI score were evaluated at $3^{\text {rd }}$ month (M3), $6^{\text {th }}$ month (M6) and $12^{\text {th }}$ month (M12). During follow-up, 13 patients lost follow-up without any assessment, 6 patients withdrew the informed consents, and those 19 patients were excluded from the final analysis. Therefore, 90 patients were included in the final analysis: 47 cases in the ABN+MTX group and 43 cases in the control group.

\section{Efficacy assessment}

The primary outcome of efficacy was DAS28-ESR remission rate, defined as the percentage of patients with DAS28-ESR $\leq 2.6$ after treatment. The secondary outcome of efficacy included DAS28-ESR response rate (defined as the percentage of patients with an improvement of DAS28-ESR $>1.2$ after treatment), DAS28-ESR low disease activity (LDA) rate (defined as the percentage of patients with DAS28-ESR $\leq 3.2$ after treatment) and the improvements of TJC, SJC, CRP level, ESR level, pain VAS score, PGA score, PhGA score, HAQ-DI score, DAS28-CRP score and DAS28-ESR score. The DAS28-ESR remission rate, DAS28-ESR response rate and DAS28-ESR LDA rate were assessed at M6 and M12. The changes of TJC, SJC, CRP level, ESR level, pain VAS score, PGA score, PhGA score, HAQ-DI score, DAS28-CRP score and DAS28-ESR score was calculated at M3, M6 and M12.

\section{Direct, indirect and total costs calculation}

Direct costs consisted of drug costs and other medical costs. And drug costs for the patients were calculated according to unit cost and the dosages recorded in the case report form (CRF). Other medical costs included outpatient cost, emergency cost and hospitalization cost. Indirect costs included lost productivity costs of patients and caregivers due to working days lost, and calculated by the losing working days multiplying the local average daily wage. Total costs were defined as the sum of drug costs, other medical costs and indirect costs. All unit costs of medication, outpatient, emergency and hospitalization were obtained from the electronic medical records of ZhuZhou Central Hospital.

\section{Pharmacoeconomic assessment}

Cost-effectiveness analysis was used for pharmacoeconomic assessment. The total costs and quality adjusted life-year (QALY) was calculated, and the difference of incremental cost-effectiveness ratio (ICER) between two groups was evaluated by incremental cost divided by incremental QALY [12]. The QALY was defined as the study we published previously[12], which were estimated form a relation function between HAQ-DI scores and Europe 
Quality of Life five-dimension (EQ-5D) questionnaire utility values [13]: EQ-5D=0.9567-0.309*HAQ-DI. The threshold of acceptable cost-effectiveness was ICER lower than three times of the annual gross domestic product (GDP) per capita, which was defined referring to the willingness-to-pay (WTP) recommended by the World Health Organization's (WHO) Choosing Interventions that are Cost-Effective (CHOICE) program [14]. In case of China, GDP per capita was 53980 RMB ( $¥)$ in $2016, ¥ 59660$ in $2017, ¥ 66006$ in $2018, ¥ 70892$ in 2019 and the average of GDP per capita from 2016 to 2019 was $¥ 62634$.

\section{Sensitivity analyses}

To evaluate the uncertainty of market effects on direct or indirect medical costs, the drug price (ABN), other medical cost and indirect cost were varied in sensitivity analyses as follows: (1) fell by $20 \%$ and $50 \%$ of their basecase prices; (2) rose by $20 \%$ and $50 \%$ of their base-case prices. Besides, the uncertainty of improvement for HAQDI score on cost-effectiveness was also performed by the sensitivity analyses, which was carried out as follows:

(1) fell by $20 \%$ and $50 \%$ of HAQ-DI score in ABN+MTX group; (2) rose by $20 \%$ and $50 \%$ of HAQ-DI score in ABN+MTX group.

\section{Statistical analysis}

Statistical analysis was performed using SPSS 21.0 statistical software (IBM, USA), and the figure was made with the use of GraphPad Prism 6.01 software (GraphPad Software Inc, USA). Data were presented as mean \pm standard deviation (SD) or count (percentage). Comparison between two groups was determined by Student's t-test or Chisquare test. All tests were 2 -sided, and $P$ value $<0.05$ was considered as statistically significant.

\section{Results}

\section{Study flow}

A total of 211 RA patients were invited to take part in our study, 48 of them were excluded (including 25 patients who missed the invitation, 15 patients who disagreed to participate and 8 patients who were unable to participate due to the distance). The remaining 163 RA patients were screened for eligibility, and 54 of them who either did not meet the inclusion criteria or met the exclusion criteria were excluded from our study. Therefore, 109 RA patients were enrolled and assigned to the $A B N+M T X$ group and the control group. In the $A B N+M T X$ group ( $N=55)$, patients received $A B N+M T X$ for 24 weeks and were subsequently followed-up for 12 months after the initiation of treatment. During the follow-up period, 8 patients were excluded (including 6 patients who lost follow-up without any assessment and 2 patients who withdrew the informed consent); finally, 47 RA patients in the ABN+MTX group were included in the efficacy and pharmacoeconomic analyses. In the control group ( $N=54)$, patients received cDMARDs for 24 weeks and were also followed-up for 12 months after the initiation of treatment. During the follow-up period, 11 patients were excluded (including 7 patients who lost follow-up without any assessment and 4 patients who withdrew the informed consent); finally, 43 RA patients in the control group were included in the efficacy and pharmacoeconomic analyses (Fig. 1).

\section{Clinical characteristics at baseline}


The mean age of RA patients was $55.5 \pm 13.0$ years in the $A B N+M T X$ group and $58.3 \pm 8.6$ years in the control group. There were 37 (78.7\%) females and 10 (21.3\%) males in the ABN+MTX group; meanwhile there were 35 $(81.4 \%)$ females as well as $8(18.6 \%)$ males in the control group. The mean disease duration was $5.2 \pm 3.3$ years in the ABN+MTX group and $4.9 \pm 2.4$ years in the control group. The mean CRP, ESR and DAS28-ESR score were $39.5 \pm 39.1 \mathrm{mg} / \mathrm{L}, 60.4 \pm 34.5 \mathrm{~mm} / \mathrm{h}$ and $5.3 \pm 0.9$, respectively in the $A B N+M T X$ group, and were $42.8 \pm 31.6 \mathrm{mg} / \mathrm{L}$, $62.6 \pm 37.9 \mathrm{~mm} / \mathrm{h}$ and $5.1 \pm 1.0$, respectively in the control group. The numbers of patients with moderate and severe disease activity were 15 (31.9\%) and 32 (68.1\%) in the ABN+MTX group, and were 18 (41.9\%) as well as 25 (58.1\%) in the control group. The detailed baseline characteristics were shown in Table 1. Notably, no difference was observed in any of the clinical characteristics between the two groups (all $P>0.05$ ). 
Table 1

Baseline characteristics

\begin{tabular}{|c|c|c|c|}
\hline Items & $A B N+M T X$ group $(N=47)$ & $\begin{array}{l}\text { Control group } \\
(\mathrm{N}=43)\end{array}$ & $P$ value \\
\hline Age (years), mean $\pm S D$ & $55.5 \pm 13.0$ & $58.3 \pm 8.6$ & 0.247 \\
\hline Gender, No. (\%) & & & 0.752 \\
\hline Female & $37(78.7)$ & $35(81.4)$ & \\
\hline Male & $10(21.3)$ & $8(18.6)$ & \\
\hline Disease duration (years), mean $\pm S D$ & $5.2 \pm 3.3$ & $4.9 \pm 2.4$ & 0.584 \\
\hline Disease activity, No. (\%) & & & 0.328 \\
\hline Moderate & 15 (31.9) & $18(41.9)$ & \\
\hline Severe & $32(68.1)$ & $25(58.1)$ & \\
\hline$T J C$, mean $\pm S D$ & $10.0 \pm 5.5$ & $9.3 \pm 5.5$ & 0.588 \\
\hline SJC, mean $\pm S D$ & $7.4 \pm 5.3$ & $6.8 \pm 4,9$ & 0.547 \\
\hline $\mathrm{CRP}(\mathrm{mg} / \mathrm{L})$, mean $\pm \mathrm{SD}$ & $39.5 \pm 39.1$ & $42.8 \pm 31.6$ & 0.660 \\
\hline $\operatorname{ESR}(\mathrm{mm} / \mathrm{h})$, mean $\pm S D$ & $60.4 \pm 34.5$ & $62.6 \pm 37.9$ & 0.772 \\
\hline Pain VAS score, mean $\pm S D$ & $6.9 \pm 1.9$ & $7.0 \pm 1.5$ & 0.865 \\
\hline PGA score, mean $\pm S D$ & $6.9 \pm 2.0$ & $7.3 \pm 1.3$ & 0.331 \\
\hline PhGA score, mean \pm SD & $6.7 \pm 1.9$ & $7.1 \pm 1.3$ & 0.246 \\
\hline HAQ-DI score, mean $\pm S D$ & $1.6 \pm 0.6$ & $1.8 \pm 0.5$ & 0.199 \\
\hline DAS28-CRP score, mean $\pm S D$ & $4.6 \pm 0.9$ & $4.6 \pm 0.9$ & 0.776 \\
\hline DAS28-ESR score, mean $\pm S D$ & $5.3 \pm 0.9$ & $5.1 \pm 1.0$ & 0.518 \\
\hline \multicolumn{4}{|l|}{ History of treatment, No. (\%) } \\
\hline cDMARDs & $29(61.7)$ & $26(60.5)$ & 0.904 \\
\hline Chinese herb & $14(29.8)$ & $11(25.6)$ & 0.656 \\
\hline Glucocorticoid & $8(17.0)$ & $6(14.0)$ & 0.688 \\
\hline NSAIDs & $10(21.3)$ & $11(25.6)$ & 0.630 \\
\hline \multicolumn{4}{|c|}{$\begin{array}{l}\text { Comparison was determined by Student's t-test or Chi-square test. ABN, anbainuo; MTX, methotrexate; SD, } \\
\text { standard deviation; TJC, tender joint count; SJC, swollen joint count; CRP, C-reactive protein; ESR, erythrocyte } \\
\text { sedimentation rate; VAS, visual analogue scale; PGA, patient global assessment; PhGA, physician global } \\
\text { assessment; HAQ-DI, Health Assessment Questionnaire Disability Index; DAS28-CRP, disease activity score } \\
\text { with } 28 \text { joints based on CRP; DAS28-ESR, disease activity score with } 28 \text { joints based on ESR; CDMARDs, } \\
\text { conventional disease-modifying antirheumatic drugs; NSAIDs, non-steroidal anti-inflammatory drugs. }\end{array}$} \\
\hline
\end{tabular}

\section{Treatment efficacy}


The change of DAS28-ESR (Fig. 2A), DAS28-CRP (Fig. 2B), SJC (Fig. 2D), pain VAS (Fig. 2E), PGA (Fig. 2F), PhGA

(Fig. 2G) and HAQ-DI (Fig. 2H) were more dominating in the ABN+MTX group compared to the control group at M3, M6 and M12 (all $P<0.05$ ). The change of TJC (Fig. 2C) was more obvious in the ABN+MTX group compared to the control group at M3 and M6 (both $P<0.05$ ). However, the change of ESR (Fig. 2I) or CRP (Fig. 2J) were similar between the two groups at each visit (all $P>0.05$ ). Moreover, for DAS28-ESR response rate, no difference was found between the ABN+MTX group and the control group at M6 $(P=0.101)$ or M12 $(P=0.194)$ (Fig. 3A). Both DAS28-ESR remission rate and DAS28-ESR LDA rate were increased in the ABN+MTX group compared to the control group at M12 (both $P<0.01$ ), but not at M6 (both $P>0.05)$ (Fig. 3B, Fig. 3C).

\section{Direct, indirect and total costs}

Drug cost was increased in the ABN+MTX group compared to the control group at M6 ( $¥ 26657.1 \pm 5877.9$ vs. $¥ 3097.2 \pm 1786.1)$ and $M 12$ ( $¥ 38433.3 \pm 14696.6$ vs. $¥ 9964.3 \pm 2281.9)$ (both $P<0.001)$. Other medical cost was also enhanced in the ABN+MTX group compared to the control group at M6 ( $¥ 11036.4 \pm 7833.5$ vs. $¥ 7980.0 \pm 2233.8$ ) $(P=0.013)$ and $M 12(¥ 18306.1 \pm 13726.5$ vs. $¥ 13641.1 \pm 1281.1)(P=0.025)$. The indirect cost was similar between the two groups at M6 ( $¥ 5184.3 \pm 5753.5$ vs. $¥ 3690.4 \pm 1962.4)$ and M12 ( $¥ 7076.0 \pm 8563.4$ vs. $¥ 7693.2 \pm 732.1)$ (both $P>0.05)$. After calculation, the total cost was higher in the ABN+MTX group than the control group at M6 ( $¥ 42877.8 \pm 13652.3$ vs. $¥ 14767.7 \pm 3276.1$ ) and M12 ( $¥ 63815.4 \pm 26520.6$ vs. $¥ 31298.6 \pm 2821.3$ ) (both $P<0.001)$ (Table 2).

Table 2

Comparison of cost between $\mathrm{ABN}+\mathrm{MTX}$ group and control group

\begin{tabular}{|c|c|c|c|c|c|c|}
\hline \multirow[t]{2}{*}{ Items } & \multicolumn{3}{|l|}{ M6 } & \multicolumn{3}{|l|}{ M12 } \\
\hline & ABN+MTX group & Control group & $\begin{array}{l}P \\
\text { value }\end{array}$ & $\mathrm{ABN}+\mathrm{MTX}$ group & Control group & $\begin{array}{l}P \\
\text { value }\end{array}$ \\
\hline $\begin{array}{l}\text { Drug } \\
\text { cost }(¥)\end{array}$ & $26657.1 \pm 5877.9$ & $3097.2 \pm 1786.1$ & $<0.001$ & $38433.3 \pm 14696.6$ & $9964.3 \pm 2281.9$ & $<0.001$ \\
\hline $\begin{array}{l}\text { Other } \\
\text { medical } \\
\text { cost ( } ¥)\end{array}$ & $11036.4 \pm 7833.5$ & $7980.0 \pm 2233.8$ & 0.013 & $18306.1 \pm 13726.5$ & $13641.1 \pm 1281.1$ & 0.025 \\
\hline $\begin{array}{l}\text { Indirect } \\
\text { cost }(¥)\end{array}$ & $5184.3 \pm 5753.5$ & $3690.4 \pm 1962.4$ & 0.099 & $7076.0 \pm 8563.4$ & $7693.2 \pm 732.1$ & 0.625 \\
\hline $\begin{array}{l}\text { Total } \\
\text { cost }(¥)\end{array}$ & $42877.8 \pm 13652.3$ & $14767.7 \pm 3276.1$ & $<0.001$ & $63815.4 \pm 26520.6$ & $31298.6 \pm 2821.3$ & $<0.001$ \\
\hline
\end{tabular}

\section{Cost-effectiveness}

The ABN+MTX group and control group presented QALY of 0.72 years and 0.48 years, respectively, thus the $A B N+M T X$ group achieved an incremental QALY of 0.24 years compared to the control group. Total cost in the $A B N+M T X$ group and control group was $¥ 63815.4$ and $¥ 31298.6$, respectively, resulting in an incremental cost of $¥ 32516.8$ in ABN+MTX group compared to control group. Based on the above data, the ABN+MTX group had an ICER of $¥ 135486.7$ per QALY compared to the control group, which was less than 3 times of mean GDP per capita 
in China during the study period (Table 3). Therefore, ABN+MTX treatment showed acceptable cost-effectiveness over cDMARDs treatment in RA patients. Moreover, cost-effectiveness analysis in subgroups was further performed. Data showed that in moderate RA patients, the ABN+MTX group generated an incremental QALY of 0.24 year and an incremental cost of $¥ 35148.1$, resulting in an ICER of $¥ 146450.4$ per QALY compared to the control group, which was less than 3 times of the mean GDP per capita in China. Meanwhile in severe RA patients, ABN+MTX group had an incremental QALY of 0.25 year and an incremental cost of $¥ 31246.8$, thus yielding an ICER of $¥ 124987.2$ per QALY compared to the control group, which was less than 2 times of the mean GDP per capita in China during the study period (Table 4). Therefore, compared to cDMARDs treatment, ABN+MTX treatment presented acceptable cost-effectiveness in moderate RA patients, and good cost-effectiveness in severe RA patients.

Table 3

Cost-effectiveness analysis

\begin{tabular}{|c|c|c|c|c|c|}
\hline Group & QALY & Incremental QALY & Total cost (¥) & Incremental cost (¥) & ICER (¥/QALY) \\
\hline$A B N+M T X$ & 0.72 & 0.24 & 63815.4 & 32516.8 & $135486.7^{*}$ \\
\hline Control & 0.48 & - & 31298.6 & - & - \\
\hline
\end{tabular}

Table 4

Cost-effectiveness analysis of subgroup

\begin{tabular}{|lllllll|}
\hline $\begin{array}{l}\text { Disease } \\
\text { activity }\end{array}$ & Group & QALY & $\begin{array}{l}\text { Incremental } \\
\text { QALY }\end{array}$ & $\begin{array}{l}\text { Total cost } \\
(¥)\end{array}$ & $\begin{array}{l}\text { Incremental cost } \\
(¥)\end{array}$ & $\begin{array}{l}\text { ICER } \\
(¥ / \text { QLY })\end{array}$ \\
\cline { 2 - 7 } & ABN+MTX & 0.77 & 0.24 & 67495.5 & 35148.1 & $146450.4^{*}$ \\
\hline Severe & Control & 0.53 & - & 32347.4 & & $124987.2^{\star *}$ \\
& ABN+MTX & 0.70 & 0.25 & 62090.3 & 31246.8 & \\
\hline
\end{tabular}

The average of gross domestic product (GDP) per capita in China from 2016 to 2019 was $¥ 62634$. * the ICER was less than 3 times of the mean GDP per capita. ${ }^{\star *}$ the ICER was less than 2 times of the mean GDP per capita. QALY, quality-adjusted life years; ICER, incremental cost-effectiveness ratio; ABN, anbainuo; MTX, methotrexate.

\section{Sensitivity analysis}

The robustness of the cost-effectiveness results was tested by sensitivity analysis, in which the main indexes were decreased or increased by $20 \%$ or $50 \%$. Data showed that: (1) Increasing ABN price by $20 \%$, decreasing ABN price by $20 \%$ and $50 \%$ yielded an ICER of $¥ 163632.1$, $¥ 105556.7$ and $¥ 54309.2$ per QALY, respectively in ABN+MTX group versus control group, which was less than 3 times, 2 times and 1 time of the mean GDP per capita in China, respectively; whereas increasing ABN price by $50 \%$ resulted in an ICER of $¥ 199487.9$ per QALY in ABN+MTX group 
versus control group, which exceeded 3 times of the mean GDP per capita in China. (2) Promoting HAQ-DI score by $20 \%$, reducing HAQ-DI score by $20 \%$ and $50 \%$ yielded an ICER of $¥ 171141.1$, $¥ 116131.4$ and $¥ 92905.1$ per QALY, respectively in ABN+MTX group versus control group, which was less than 3 times, 2 times and 2 times of the mean GDP per capita in China, respectively; while promoting HAQ-DI score by $50 \%$ resulted in an ICER of $¥ 250129.2$ per QALY in ABN+MTX group versus control group, which exceeded 3 times of the mean GDP per capita in China. (3) Regarding other medical cost and indirect cost, increasing or decreasing these two indexes by $20 \%$ or $50 \%$ all yielded in ICERs that did not exceed 3 times of the mean GDP per capita in China. These data indicated that ABN price and HAQ-DI score, but not other medical cost or indirect cost, impacted the costeffectiveness of $A B N+M T X$ treatment versus CDMARDs treatment greatly, and ABN+MTX treatment generally exhibited superior cost-effectiveness to cDMARDs treatment in RA patients in China. (Table 5). In addition, we used different utility formulas to calculate the cost-effectiveness, and found that ABN+MTX treatment was costeffective than cDMARDs treatment based on all these formulas (Supplementary Table 1[13, 15-18]). 
Table 5

Sensitivity analysis

Items

QALY Incrementa

QALY $\quad(¥) \quad$ cost $(¥) \quad(¥ / \mathrm{QALY})$

\section{Drug price}

Price of ABN up by $20 \%$

$A B N+M T X$

0.72

0.24

0.48

70570.3

39271.7

Control

Price of $A B N$ up by $50 \%$

ABN+MTX

0.72

0.24

0.48

31298.6

Control

Price of $A B N$ down by $20 \%$

$105556.7^{\star \star}$

$\mathrm{ABN}+\mathrm{MTX}$

0.72

0.24

$56632.2 \quad 25333.6$

Control

0.48

31298.6

Price of $A B N$ down by $50 \%$

$A B N+M T X$

0.72

0.24

0.48

79175.7

47877.1

31298.6

199487.9

Control

\section{Other medical cost}

Other medical cost up by $20 \%$

$A B N+M T X$

$0.72 \quad 0.24$

$67476.6 \quad 33449.8$

Control

0.48

34026.8

Other medical cost up by $50 \%$

$A B N+M T X$

$0.72 \quad 0.24$

72968.5

38119.2

Control

0.48

Other medical cost down by $20 \%$

$131599.2^{\star}$

$\mathrm{ABN}+\mathrm{MTX}$

0.72

0.24

Control

0.48

$60154.2 \quad 31583.8$

$28570.4 \quad-$

Other medical cost down by $50 \%$

$A B N+M T X$

0.72

0.24

0.48

54662

Control

0.48

Indirect cost

$163632.1^{*}$

$54309.2^{\star \star *}$

$139374.2^{*}$

$145205.5^{\star}$

34849.3 


$\begin{array}{lllll}\mathrm{ABN}+\mathrm{MTX} & 0.72 & 0.24 & 65230.6 & 32393.3 \\ \text { Control } & 0.48 & - & 32837.3 & -\end{array}$

Indirect cost up by $50 \%$

$134200.8^{*}$

$\begin{array}{lllll}\mathrm{ABN}+\mathrm{MTX} & 0.72 & 0.24 & 67353.4 & 32208.2 \\ \text { Control } & 0.48 & - & 35145.2 & -\end{array}$

Indirect cost down by $20 \%$

$136000.8^{*}$

$\begin{array}{lllll}\mathrm{ABN}+\mathrm{MTX} & 0.72 & 0.24 & 62400.2 & 32640.2 \\ \text { Control } & 0.48 & - & 29760.0 & -\end{array}$

Indirect cost down by $50 \%$

$136772.5^{\star}$

ABN+MTX

$0.72 \quad 0.24$

$60277.4 \quad 32825.4$

Control

0.48

27452.0

\section{HAQ-DI score change for ABN+MTX group}

HAQ-DI score up by $20 \%$

$\begin{array}{llllll}\text { ABN+MTX } & 0.67 & 0.19 & 63815.4 & 32516.8 & 171141.1^{*} \\ \text { Control } & 0.48 & - & 31298.6 & - & -\end{array}$

HAQ-DI score up by $50 \%$

$\begin{array}{llllll}\text { ABN+MTX } & 0.61 & 0.13 & 63815.4 & 32516.8 & 250129.2 \\ \text { Control } & 0.48 & - & 31298.6 & - & \end{array}$

HAQ-DI score down by $20 \%$

\begin{tabular}{lccccc} 
ABN+MTX & 0.76 & 0.28 & 63815.4 & 32516.8 & $116131.4^{* *}$ \\
Control & 0.48 & - & 31298.6 & - & - \\
\hline HAQ-DI score down by 50\% & & & & & \\
\hline ABN+MTX & 0.83 & 0.35 & 63815.4 & 32516.8 & $92905.1^{* *}$ \\
Control & 0.48 & - & 31298.6 & - & -
\end{tabular}

The average of gross domestic product (GDP) per capita in China from 2016 to 2019 was $¥ 62634$. * the ICER was less than 3 times of the mean GDP per capita. ${ }^{* \star}$ the ICER was less than 2 times of the mean GDP per capita. ${ }^{\star \star \star}$ the ICER was less than 1 times of the mean GDP per capita. QALY, quality-adjusted life years; ICER, incremental cost-effectiveness ratio; ABN, anbainuo; MTX, methotrexate; HAQ-DI, Health Assessment Questionnaire Disability Index. 


\section{Discussion}

In the present study, we found that: (1) compared to cDMARDs treatment, ABN+MTX treatment showed acceptable cost-effectiveness in RA patients, and subgroup analysis revealed that it presented acceptable cost-effectiveness in moderate RA patients as well as good cost-effectiveness in severe RA patients; (2) the cost-effectiveness of $A B N+M T X$ treatment compared to cDMARDs treatment presented relative robustness to the turbulence of $A B N$ price and HAQ-DI score.

Since the appearance of TNF inhibitors on the market, the prognosis of RA patients has been improved quite a lot [6]. TNF inhibitors present great efficacy in controlling the disease progression by suppressing inflammation and disease activity, reducing radiology progression to some extent as well as improving the quality of life of RA patients [7]. However, TNF inhibitors are quite costly. It is estimated that the annual drug cost of TNF inhibitors reaches around $\$ 36000$ in the United States [3]. Moreover, according to a previous study in Poland, the average drug cost for patients who received etanercept for 24 months was 68514 PLN [19]; Therefore, the costeffectiveness of TNF inhibitors should be carefully assessed to support the guidance of TNF inhibitors administration in RA patients. Regarding the cost-effectiveness of TNF inhibitors, a previous study finds that TNF inhibitors are more cost-effective compared with rituximab, a CD20 monoclonal antibody, in RA patients in Finland [20]. Another study shows that adalimumab (a TNF inhibitor) is cost-effective compared to cDMARDs in RA patients in German [21]. However, most of the studies are performed in developed countries, and the assessments of cost-effectiveness of TNF inhibitors in Chinese RA patients are relatively deficient and are urgently needed.

$\mathrm{ABN}$ is a novel biosimilar to etanercept independently developed by a Chinese pharmacology company. One phase II trial shows that after the administration of ABN and MTX combination for 24 weeks, the proportions of patients who achieved ACR50 and ACR70 are $53.6 \%$ and $27.7 \%$, respectively, which are significantly higher than monotherapy of ABN or MTX [10]. Another phase III trial reveals that the ABN+MTX administration for 12 weeks promotes ACR20, ACR50 and ACR70 rate in MTX-inadequately responded RA patients compared to placebo; meanwhile $A B N$ is well-tolerated in those patients [11]. Considering that $A B N$ presents good treatment efficacy as well as lower price than original etanercept, it might be vital to evaluate the cost of $A B N+M T X$ versus cDMARDs, further investigating their comparison of cost-effectiveness. In the present study, ABN+MTX treatment had generated higher drug cost and other medical cost than cDMARDs treatment, resulting in a higher total cost, meanwhile, drug cost (mainly cost on $A B N$ ) was the dominating cost. Moreover, we found that the indirect cost in the $A B N+M T X$ group was numerically lower than the that in control group at M12, which could be explained by that: ABN+MTX had better treatment effect than CDMARDs in RA patients (mentioned above), which might improve the quality of life of the patients, thus saving the cost caused by loss of productivity of patients or caregivers due to loss of working days. These data indicated that $\mathrm{ABN}+\mathrm{MTX}$ treatment was more costly compared to cDMARDs treatment, and to evaluate the cost-effectiveness in ABN+MTX treatment versus cDMARDs treatment was quite important.

The evaluation on the cost-effectiveness of TNF inhibitors is quite popular in developed countries, whereas it is relatively deficient in China and only a few studies have been conducted before. One previous study reveals that etanercept+MTX treatment is not cost-effective compared to triple cDMARDs treatment with an ICER of $¥ 2,939,506.7$ per QALY in treating Chinese RA patients [22]. Meanwhile, our pilot study shows that ABN+MTX treatment presents acceptable cost-effectiveness compared to cDMARDs treatment in a small-sample-sized Chinese RA patients [12]. In order to further verify the results in our pilot study, we conducted the present study and found that the ICER of ABN+MTX treatment compared to cDMARDs treatment was $¥ 135486.7$ per QALY, which 
was less than the cost-effectiveness threshold. Notably, the cost-effectiveness of ABN+MTX treatment compared to cDMARDs treatment was more obvious in severe RA patients, which was less than 2 times of the mean GDP per capita in China. Possible explanations might be that: (1) the therapeutic efficacy of ABN+MTX treatment was better in severe RA patients, which might cause a promoted incremental QALY and reduced ICER; (2) the good treatment effect of $A B N+M T X$ reduced the disease activity in severe RA patients, which might decrease the hospitalization cost, thus reducing the total cost in patients received $A B N+M T X$. Therefore, $A B N+M T X$ treatment presented cost-effectiveness that was more obvious in severe RA patients, and it might be more plausible to administrate $A B N+M T X$ in severe RA patients.

The sensitivity analysis in this study revealed that ABN cost and HAQ-DI score in the ABN+MTX group were two predominant factors that affected the cost-effectiveness of ABN+MTX treatment versus cDMARDs treatment. Possible explanations might be that: ABN price was much higher than other medical cost and indirect cost (as shown in the cost analysis), thus ABN price greatly affected total cost and predominantly impacted the costeffectiveness of $A B N+M T X$ treatment versus cDMARDs treatment. (2) HAQ-DI score was a dominating factor that impacts the QALY, thus it also greatly affected the cost-effectiveness of ABN+MTX treatment versus cDMARDs treatment. Furthermore, increasing the price of $A B N$ or reducing HAQ-DI score in the ABN+MTX group by $20 \%$ still showed acceptable cost-effectiveness. These data indicated that ABN+MTX treatment versus cDMARDs treatment remained acceptable cost-effectiveness under the premise of the turbulence of ABN price and HAQ-DI score in RA patients [3].

There were several limitations in this study. Firstly, we did not perform the patients' survey to investigate the acceptability curve analysis and evaluate the cost-effectiveness within a range of cost-effectiveness thresholds due to insufficient manpower and fund. Instead, the cost-effectiveness threshold was simply set as 3 times of the mean GDP per capita in China during this study, which might cause bias; and the acceptability analysis could be investigated further. Secondly, due to the imbalanced development between south part and north part, as well as coastal area and inner land of China, the cost-effectiveness threshold might be unaffordable in some relatively undeveloped regions. Therefore, further studies should be conducted to evaluate the cost-effectiveness of ABN+MTX treatment in different regions of China. Thirdly, the sample size in this study was relatively small, and studies with larger sample sizes should be further conducted to verify our results. Finally, the follow-up duration of this study was relatively short, and the long-term cost-effectiveness of ABN+MTX treatment in RA patients was unclear, which could be investigated further.

Collectively, ABN+MTX treatment exhibits acceptable cost-effectiveness compared to cDMARDs treatment in Chinese RA patients, meanwhile, its cost-effectiveness presents some robustness to the turbulence of ABN price and HAQ-DI score.

\section{Declarations}

\section{Ethics approval and consent to participate}

All procedures were conducted in accordance with the provisions of the Declaration of Helsinki and Good Clinical Practice guidelines as defined by the International Conference on Harmonization. All patients signed the written informed consents. Besides, the study was registered on the Chinese Clinical Trial Registry (ChiCTR) affiliated to World Health Organization (WHO) (www.chictr.org.cn) with registry number ChiCTR2000032534. 


\section{Consent for Publication}

Not applicable.

\section{Availability of data and materials}

All data generated or analysed during this study are included in this published article [and its supplementary information files].

\section{Competing interests}

The authors declare that they have no competing interests.

\section{Funding}

This study was supported by Provincial Natural Science Foundation of HuNan (No.2017JJ4069).

\section{Authors' contributions}

Feng Tian and Zhenhua Wen made substantial contributions to the design of the present study. Data acquisition was performed by Feng Tian, Zhenhua Wen, Jingyang Li, Xiaowen Luo, Li Deng, Liang Zhang, Jingyun He, Fangling Yao and Zheng Liao. Data analysis and interpretation was performed by Feng Tian, Zhenhua Wen and Jingyang Li. Feng Tian, Zhenhua Wen and Jingyang Li critically revised the manuscript for important intellectual content. All authors approved the final version of the manuscript. All authors agree to be accountable for all aspects of the work in ensuring that questions related to the accuracy or integrity of the work are appropriately investigated and resolved.

\section{Acknowledgements}

Not applicable.

\section{References}

1. Smolen JS, et al. Rheumatoid arthritis. Nat Rev Dis Primers. 2018;4:18001.

2. Wang G, Mu R, Xu H. Management of rheumatoid arthritis in People's Republic of China - focus on tocilizumab and patient considerations. Int J Gen Med. 2015; 8187 - 94.

3. Aletaha D, Smolen JS. Diagnosis and Management of Rheumatoid Arthritis: A Review. JAMA. 2018;320(13):1360-72.

4. Dougados M. Comorbidities in rheumatoid arthritis. Curr Opin Rheumatol. 2016;28(3):282-8.

5. Fazal SA, et al. A Clinical Update and Global Economic Burden of Rheumatoid Arthritis. Endocr Metab Immune Disord Drug Targets. 2018;18(2):98-109. 
6. Smolen JS, et al. EULAR recommendations for the management of rheumatoid arthritis with synthetic and biological disease-modifying antirheumatic drugs: 2016 update. Ann Rheum Dis. 2017;76(6):960-77.

7. Goodman SM. Rheumatoid arthritis: Perioperative management of biologics and DMARDs. Semin Arthritis Rheum. 2015;44(6):627-32.

8. Kay J, et al. Consensus-based recommendations for the use of biosimilars to treat rheumatological diseases. Ann Rheum Dis. 2018;77(2):165-74.

9. $\mathrm{Hu} \mathrm{H}$, et al. Burden of rheumatoid arthritis from a societal perspective: A prevalence-based study on cost of this illness for patients in China. Int J Rheum Dis. 2018;21(8):1572-80.

10. Chen XX, et al. A multicenter, randomized, double-blind clinical trial of combination therapy with Anbainuo, a novel recombinant human TNFRII:Fc fusion protein, plus methotrexate versus methotrexate alone or Anbainuo alone in Chinese patients with moderate to severe rheumatoid arthritis. Clin Rheumatol. 2013;32(1):99-108.

11. Chen XX, et al. A randomized, controlled trial of efficacy and safety of Anbainuo, a bio-similar etanercept, for moderate to severe rheumatoid arthritis inadequately responding to methotrexate. Clin Rheumatol. 2016;35(9):2175-83.

12. Tian F, et al. A novel etanercept biosimilar Anbainuo plus methotrexate exhibits increased cost-effectiveness compared to conventional disease-modifying anti-rheumatic drugs in treating rheumatoid arthritis patients. Med (Baltim). 2019;98(48):e17750.

13. Carreno A, et al. Using HAQ-DI to estimate HUI-3 and EQ-5D utility values for patients with rheumatoid arthritis in Spain. Value Health. 2011;14(1):192-200.

14. Cost effectiveness and strategic planning (WHO-CHOICE) [M].

15. Spalding JR, Hay J. Cost effectiveness of tumour necrosis factor-alpha inhibitors as first-line agents in rheumatoid arthritis. Pharmacoeconomics. 2006;24(12):1221-32.

16. Ashraf RBNST. UT3 ESTIMATING HEALTH UTILITY FROM A PHYSICAL FUNCTION ASSESSMENT. IN RHEUMATOID ARTHRITIS (RA) PATIENTS TREATED WITH ADALIMUMAB (D2E7). Value in Health. 2002;5(6):452-53.

17. Ducournau PKA, Wintfeld N. Comparison of linear and non-linear utility mapping between HAQ and EQ-5D using pooled data from the tocilizumab trials OPTION and LITHE [M]. Glasgow: British Society of Rheumatology; 2009.

18. Bansback NJ, Brennan A, Ghatnekar O. Cost effectiveness of adalimumab in the treatment of patients with moderate to severe rheumatoid arthritis in Sweden. Ann Rheum Dis. 2005;64(7):995-1002.

19. Burmester GR, et al. Tocilizumab in early progressive rheumatoid arthritis: FUNCTION, a randomised controlled trial. Ann Rheum Dis. 2016;75(6):1081-91.

20. Huoponen S, et al. Cost-effectiveness of abatacept, tocilizumab and TNF-inhibitors compared with rituximab as second-line biologic drug in rheumatoid arthritis. PLoS One. 2019;14(7):e0220142.

21. Gissel C, Gotz G, Repp H. Cost-effectiveness of adalimumab for rheumatoid arthritis in Germany. Z Rheumatol. 2016;75(10):1006-15.

22. Shi ZC, Fei HP, Wang ZL. Cost-effectiveness analysis of etanercept plus methotrexate vs triple therapy in treating Chinese rheumatoid arthritis patients. Med (Baltim). 2020;99(3):e16635. 


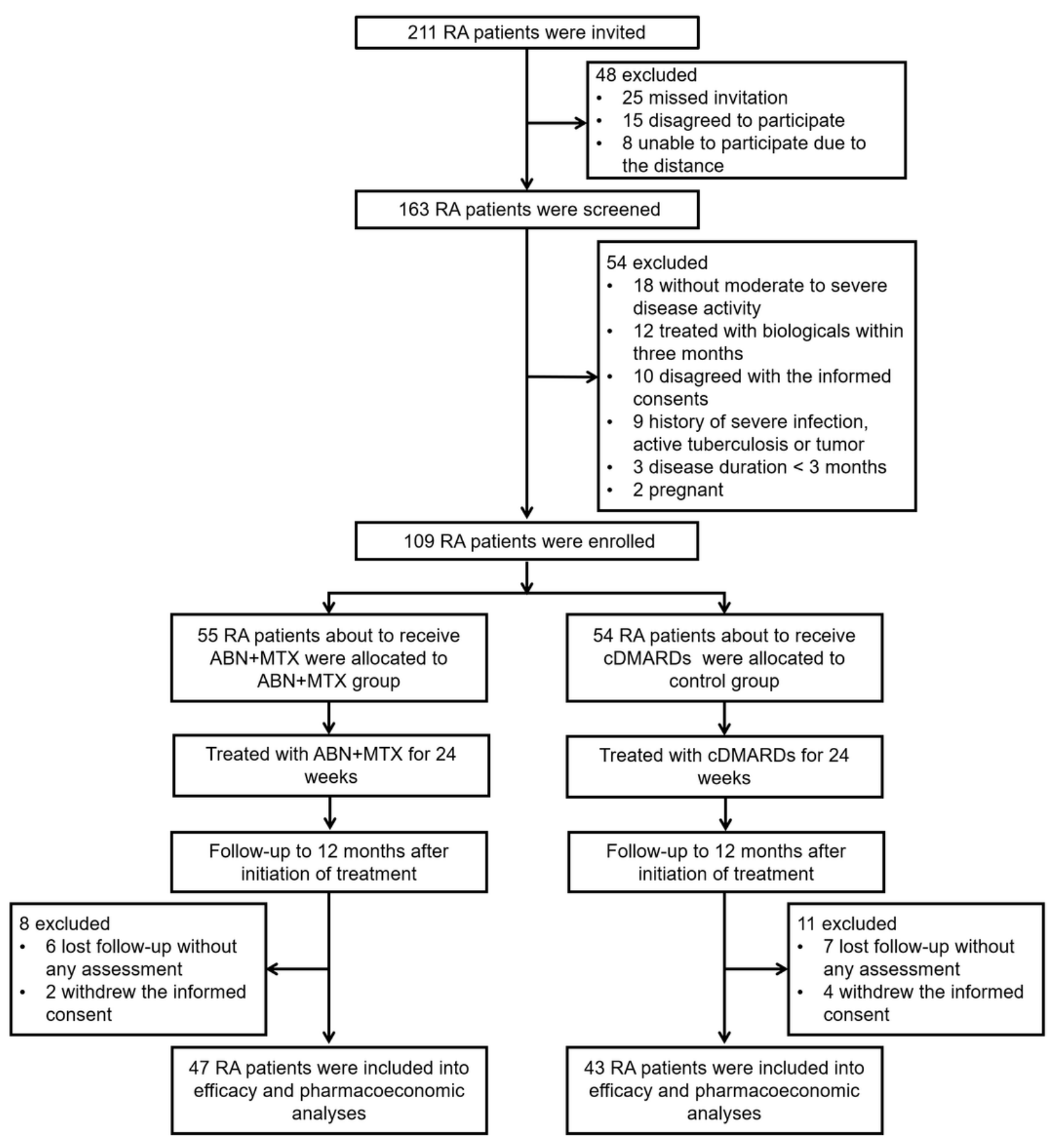

\section{Figure 1}

Study flow chart. RA: rheumatoid arthritis; ABN: anbainuo; MTX: methotrexate; cDMARDs: conventional diseasemodifying antirheumatic drugs. 
A

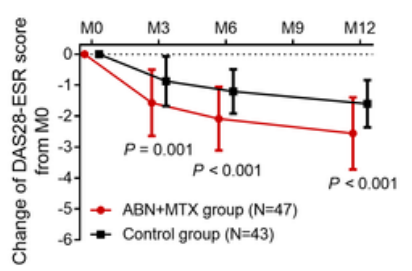

E

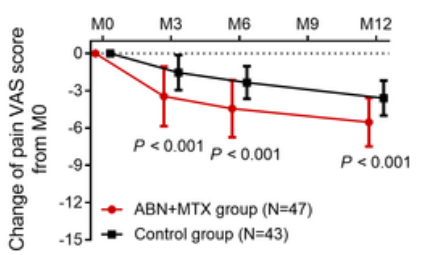

I

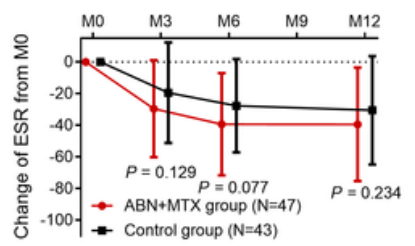

B

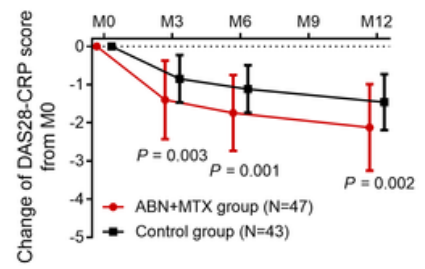

F

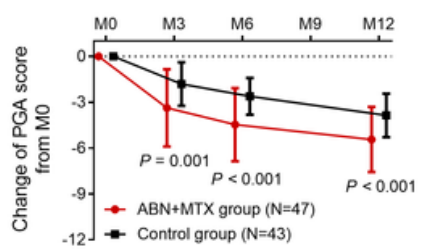

J

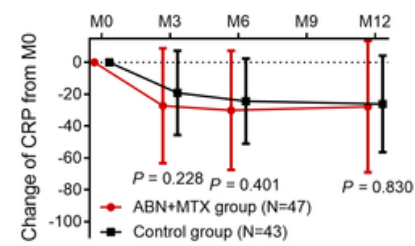

C

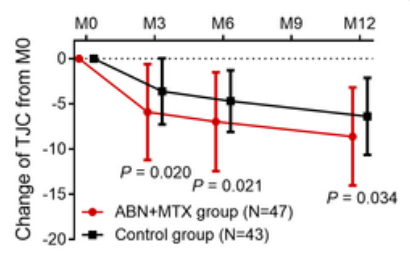

G

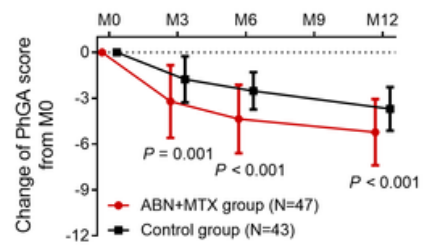

D

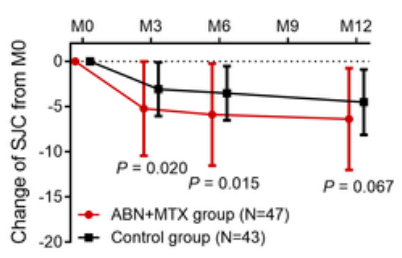

$\mathrm{H}$

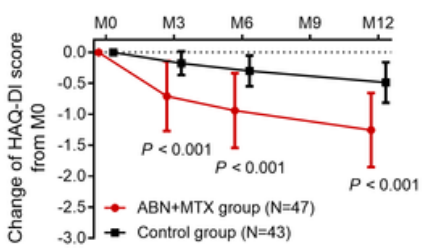

Figure 2

Comparison of improvement of DAS28-ESR (A), DAS28-CRP (B), TJC (C), SJC (D) pain VAS (E), PGA (F), PhGA (G), HAQ-DI (H), ESR (I), and CRP (J) between the ABN+MTX group and the control group. ESR: erythrocyte sedimentation rate; CRP: C-reactive protein; DAS28-ESR: disease activity score with 28 joints based on ESR; DAS28-CRP: disease activity score with 28 joints based on CRP; TJC: tender joint count; SJC: swollen joint count; VAS: visual analogue scale; PGA: patient global assessment; PhGA: physician global assessment; HAQ-DI: health assessment questionnaire disability index; ABN: anbainuo; MTX: methotrexate; M0: baseline; M3: 3rd month; M6: 6th month; M9: 9th month; M12: 12th month.

A

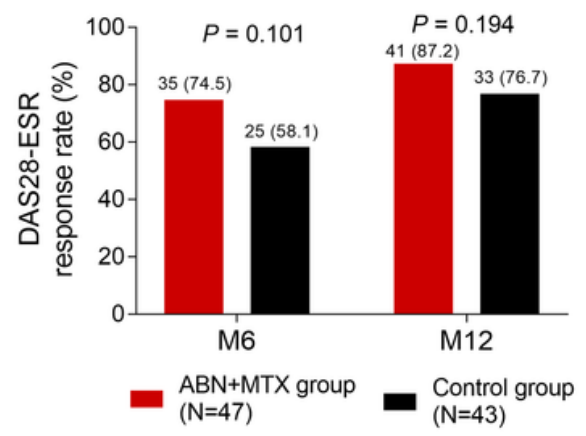

$\mathrm{B}$

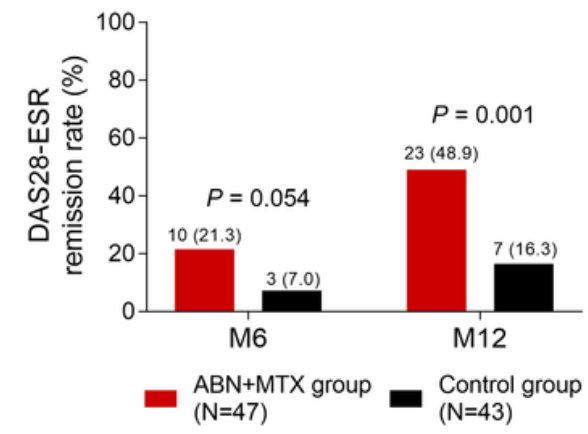

C

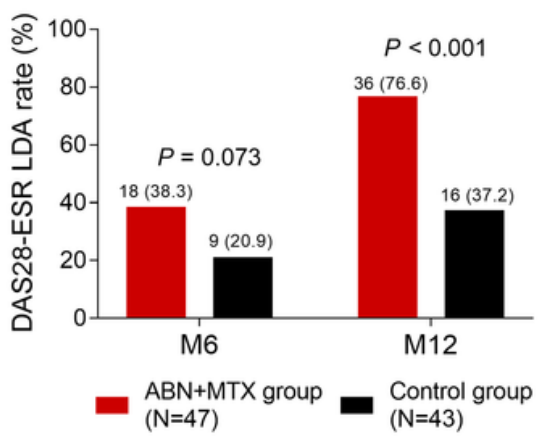

Figure 3

Comparison of DAS28-ESR response rate (A), DAS28-ESR remission rate (B), DAS28-ESR LDA rate (C) between the $A B N+M T X$ group and the control group. DAS28-ESR: disease activity score with 28 joints based on erythrocyte sedimentation rate; LDA: Iow disease activity; ABN: anbainuo; MTX: methotrexate; M6: 6th month; M12: 12th month. 


\section{Supplementary Files}

This is a list of supplementary files associated with this preprint. Click to download.

- Supplementarytable1.docx 